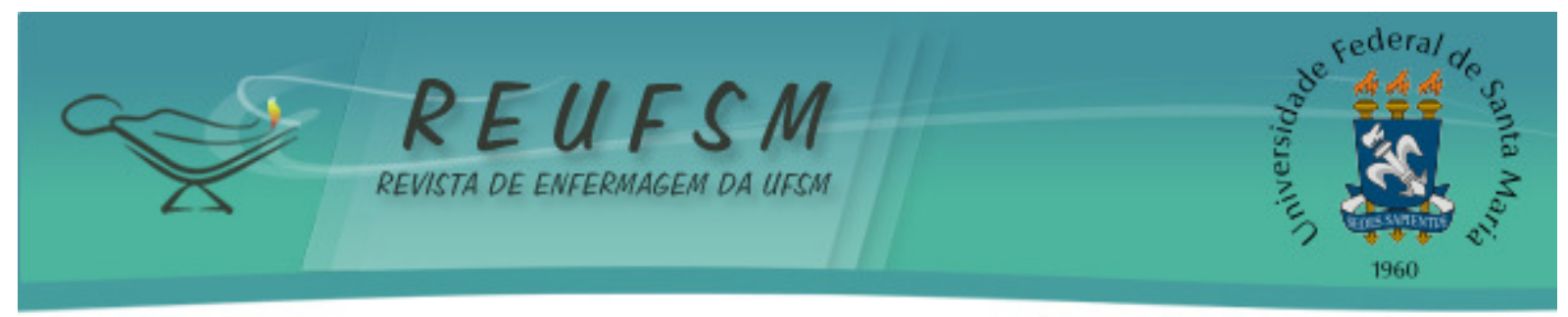

ARTIGO ORIGINAL

\title{
REDES DE ATENÇÃO À SAÚDE: PERCEPÇÃO DE USUÁRIOS DE UMA UNIDADE DE SAÚDE DA FAMÍLIA
}

\section{HEALTH NETWORKS CARE: THE PERCEPTION OF FAMILY HEALTH UNIT USERS \\ REDES DE ATENCION A LA SALUD: PERCEPCIÓN DE USUARIOS DE UNA UNIDAD DE SALUD FAMILIAR}

Doi: $10.5902 / 2179769225209$

\author{
Adrielle Chermont da Silva ${ }^{1}$ \\ Maria Denise Schimith ${ }^{2}$ \\ Taís Falcão Gomes ${ }^{3}$ \\ Teresinha Heck Weiller ${ }^{4}$ \\ Gabriela Favero Alberti ${ }^{5}$ \\ Bruna Sodré Simon ${ }^{6}$
}

RESUMO: Objetivo: conhecer a percepção dos usuários de uma Unidade de Saúde da Família sobre a Rede de Atenção à Saúde. Método: trata-se de uma pesquisa qualitativa, do tipo exploratório-descritiva, realizada com seis usuários de saúde, de fevereiro a julho de 2012. Para coleta de dados utilizaram-se entrevistas semiestruturadas, que foram analisadas sob a Proposta Operativa. Resultados: identificou-se que há longo tempo de espera pelo atendimento especializado, o que leva os usuários a procurarem diferentes meios de acesso à rede. Estes receberam poucas orientações dos profissionais de saúde, que, por sua vez, demonstram pouco envolvimento e responsabilização, refletindo no modo em que os usuários percebem a unidade e os serviços prestados. Conclusões: percebeu-se que ainda existe relação vertical entre profissionais de saúde e usuários, uma vez que não foi identificado a escuta e que faltaram orientações sobre o fluxo de acesso, necessitando assim da construção de espaços acolhedores.

Descritores: Saúde da família; Atenção primária à saúde; Continuidade da assistência ao paciente; Sistema único de saúde.

ABSTRACT: Aim: to know the perception of the users of a Family Health Unit about the Health Care Network. Method: through a qualitative research approach, this is both an exploratory and a descriptive study, carried out with six health care users of the chosen unit, from February to July 2012. Semi-structured interviews were used for data collection, which were analyzed under the Operative Proposal. Results: it was identified that there is a long waiting time for specialized services, which leads users to searching for different means of accessing the network. These users received little orientation from health professionals, who in turn demonstrated little involvement and accountability,

\footnotetext{
${ }^{1}$ Enfermeira. Mestre em Enfermagem. Universidade Federal de Santa Maria. Santa Maria, RS, Brasil. E-mail: adriellechs@gmail.com

${ }^{2}$ Enfermeira. Doutora em Ciências. Professora Adjunta da Universidade Federal de Santa Maria. Santa Maria, RS, Brasil. E-mail: ma.denise2011@gmail.com

${ }^{3}$ Enfermeira. Mestre em Enfermagem. Universidade Federal de Santa Maria. Santa Maria, RS, Brasil. E-mail: taissfg@gmail.com

${ }^{4}$ Enfermeira. Doutora em Enfermagem em Saúde Pública. Professora Associada da Universidade Federal de Santa Maria. Santa Maria, RS, Brasil. E-mail: weiller2@hotmail.com

${ }^{5}$ Enfermeira. Mestre em Enfermagem. Universidade Federal de Santa Maria. Santa Maria, RS, Brasil. E-mail: g_falberti@hotmail.com

${ }^{6}$ Enfermeira. Doutoranda pelo Programa de Pós-Graduação em Enfermagem da Universidade Federal de Santa Maria. Professora Assistente da Universidade Federal do Pampa (UNIPAMPA), Campus Uruguaiana, RS, Brasil. Vice-líder do Núcleo de Estudos em Família e Cronicidade - UNIPAMPA. E-mail: enf.brusimon@gmail.com
} 


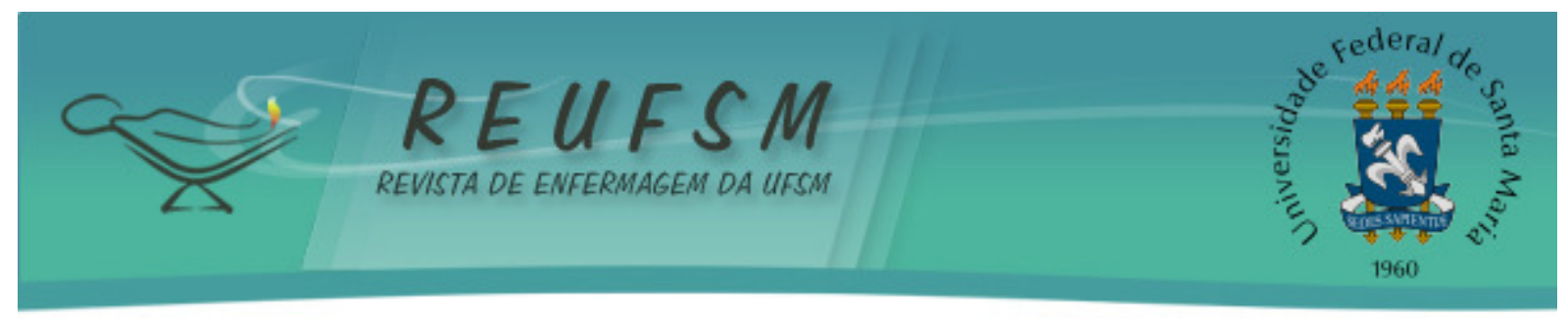

which reflects how users perceive the unit and the provided services. Conclusions: it was noticed that there is still a vertical relationship between health professionals and users, since listening was not identified and there were no guidance on access flow, which requires the construction of cozy spaces.

Descriptors: Family health; Primary health care; Continuity of patient care; Unified health system.

RESUMEN: Objetivo: conocer la percepción de los usuarios de una Unidad de Salud de la Familia, sobre la Red de Atención a la Salud. Método: se trata de una investigación cualitativa, tipo exploratoria descriptiva, realizada con seis usuarios de salud, entre febrero y julio de 2012. Para la recolección de datos se utilizó entrevistas semiestructuradas, analizadas a partir de la Propuesta Operativa. Resultados: se identificó que hay un largo tiempo de espera para atención especializada, eso lleva los usuarios a buscar diferentes medios de acceso a la red. Los usuarios recibieron pocas orientaciones de los profesionales de la salud, que a su vez demuestran poca implicación y responsabilidad, evidenciando el modo en que los usuarios perciben la unidad y los servicios prestados. Conclusiones: se percibió que aún existe relación vertical entre profesionales de salud y usuarios, una vez que no fue identificada la escucha, además de la falta de orientaciones sobre el flujo de acceso, lo que requiere la construcción de espacios acogedores.

Descriptores: Salud de la familia; Atención primaria de salud; Continuidad de la atención al paciente; Sistema único de salud.

\section{INTRODUÇÃO}

Com a finalidade de reduzir a fragmentação, fortalecer a integralidade e a descentralização da assistência em saúde, em 30 de dezembro de 2010, foi lançada a Portaria $n^{\circ} 4.279$ pelo Ministério da Saúde (MS). ${ }^{1}$ Essa portaria traça as diretrizes para estruturação das Redes de Atenção à Saúde (RAS) e objetiva melhorar o desempenho do Sistema Único de Saúde (SUS) em relação ao acesso, equidade e eficácia. ${ }^{1}$

As RAS são conjuntos de serviços de saúde que possuem uma missão única e objetivos comuns, os quais buscam agir de forma cooperativa e interdependente. ${ }^{2}$ Dessa maneira, permitem ofertar uma atenção contínua e integral a determinada população, coordenada pela Atenção Primária à Saúde (APS), a qual atua como elo entre os variados serviços de saúde e garante o cuidado por meio de uma relação horizontal, contínua e integral. ${ }^{1}$

Nesta concepção de redes, a APS é constituída por uma equipe de saúde multidisciplinar, preparada e proativa, com a responsabilidade de coordenar o cuidado e o caminho que o usuário precisa percorrer nela. ${ }^{3}$ A equipe, articulada com a população adscrita, deve considerar a pessoa em sua singularidade e inserção sociocultural, a fim de possibilitar o acesso universal e contínuo a serviços de saúde de qualidade e resolutivos.

Para isso, faz-se necessária a criação do vínculo e acolhimento entre profissionais e usuários, possibilitando uma relação de corresponsabilização, de modo a atender às necessidades de saúde do usuário. ${ }^{4}$ Nesse sentido, é importante que, além dos profissionais, os usuários também estejam envolvidos nesse processo, a fim de reforçar a interação entre gestores e cidadãos, promovendo a democracia e contribuindo na tomada de decisões de qualidade. ${ }^{5}$ Além disso, tal temática se torna importante uma vez que, no ano de 2011, a Agenda Nacional de Prioridades em Pesquisa publicou a necessidade da realização de pesquisas sobre controle social, gestão do trabalho e atenção à saúde, bem como sobre comunicação e informação nos serviços de saúde. ${ }^{6}$ 


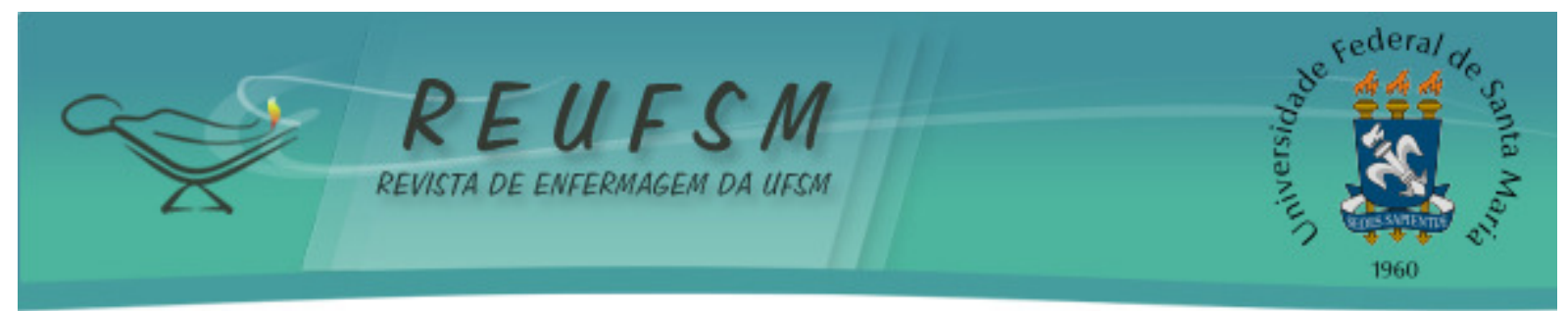

Assim, justifica-se a importância da realização deste estudo, que teve como questão de pesquisa: "como os usuários de uma Unidade de Saúde da Família (USF) percebem a Rede de Atenção à Saúde no município de Santa Maria?" e que teve como objetivo: conhecer a percepção dos usuários acerca da Rede de Atenção à Saúde no município de Santa Maria.

\section{MÉTODO}

Trata-se de uma pesquisa qualitativa do tipo exploratório-descritiva. Essa abordagem permite compreender a complexidade e as singularidades dos contextos, por meio do ponto de vista dos mais diversos atores sociais envolvidos. ${ }^{7}$

As entrevistas foram realizadas com usuários do SUS, e são provenientes do banco de dados do projeto "Relações entre trabalhadores de saúde e usuários em uma Unidade Saúde da Família (USF): a construção da rede de atenção à saúde", realizado na cidade de Santa Maria, no interior do Rio Grande do Sul, Brasil. Tal projeto fez uso de diferentes formas de coleta e contou com um período de observação, com duração total de 76h25min, entrevistas com os trabalhadores e usuários e buscas em documentos e em registros de sistemas de informações.

0 instrumento de coleta de informações utilizado para a extração de dados deste estudo foi a entrevista semiestruturada. As entrevistas foram previamente agendadas e realizadas na USF. 0 tempo médio de duração de cada entrevista foi de 30 minutos. As entrevistas foram gravadas em dispositivo digital e, posteriormente, transcritas na íntegra.

As questões básicas foram listadas em um roteiro, que abrangia a procura por atendimento e a experiência sobre a trajetória do encaminhamento, sendo as seguintes: conte-me sobre o que fez você procurar a USF? como foi a decisão de ser feito este encaminhamento? você sabe por que precisa este encaminhamento? quais as orientações recebidas do trabalhador de saúde sobre como atingir o objetivo do encaminhamento? que dificuldades enfrentará para conseguir chegar ao destino do encaminhamento para a RAS de Santa Maria? como você chegará ao destino previsto no encaminhamento? que outros caminhos? quem pode ajudar? como você chegará num período aceitável de tempo de espera para seu caso? na sua opinião/experiência, como o trabalhador da USF pode ajudar neste caminhar? depois que atingir ao objetivo do encaminhamento (consulta com especialista, exames etc.) quais serão os próximos passos?.

A coleta de dados ocorreu no período de fevereiro a julho de 2012. Para este estudo incluíram-se os usuários adultos da USF que receberam um encaminhamento a outro ponto da RAS de Santa Maria/RS e foram excluídas as gestantes e usuários com encaminhamento para serviços de urgência/emergência.

O critério para interrupção da coleta foi atingir os objetivos da pesquisa, que indica suspensão da inclusão de novos participantes quando os dados obtidos passam a apresentar, na avaliação do pesquisador, certa redundância. ${ }^{7}$

Foram realizadas seis entrevistas, analisadas sob a Proposta Operativa ${ }^{7}$, caracterizada pelo emprego de dois momentos operacionais: o primeiro inclui a fase exploratória da investigação e o segundo momento denomina-se de interpretativo. Primeiramente foram determinados os fundamentos do estudo, já na fase interpretativa ocorreu a ordenação e a classificação dos dados. ${ }^{7}$ Depois de alcançada a profunda imersão sobre o material empírico e finalizadas as etapas de organização e classificação dos dados, foi realizada a análise final, em que ocorreu uma releitura das unidades de significado, em paralelo com os objetivos da pesquisa, integrando-as com os pressupostos teóricos e com o contexto dos participantes. 


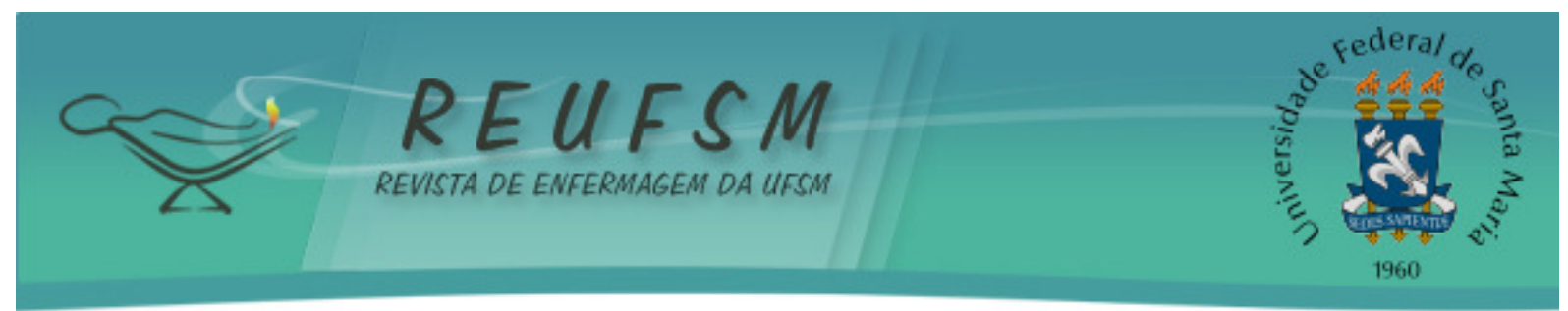

A participação na pesquisa se deu a partir da aceitação dos entrevistados e firmada por meio da assinatura do Termo de Consentimento Livre e Esclarecido. Os entrevistados tiveram garantidos seu anonimato e foram identificadas com a letra E de entrevista, seguida por uma ordem numérica. A pesquisa procurou assegurar e valorizar uma condução ética durante todo o processo de pesquisa, sendo consideradas as orientações e disposições da Resolução $n^{\circ} 196 / 96$, do $M S{ }^{8}{ }^{8}$ vigente no momento da coleta dos dados. A pesquisa foi aprovada sob protocolo número 1939/11 do Comitê de Ética em Pesquisa (CEP) da Universidade Federal de São Paulo (UNIFESP), no dia 09 de dezembro de 2011.

\section{RESULTADOS}

No processo de análise das entrevistas, surgiram duas categorias temáticas. Elas estão ilustradas com depoimentos dos participantes, além da interpretação dos resultados. Inicialmente, é apresentada a caracterização dos participantes e, posteriormente, as categorias.

\section{Caracterização dos participantes}

Todos eram do sexo feminino, com idade variável entre 34 e 72 anos. Três entrevistadas possuíam ensino fundamental incompleto, uma possuía ensino fundamental completo, uma concluiu o ensino médio e, por fim, uma apresentou segundo grau completo. Todas possuíam encaminhamento para especialistas da RAS, os quais eram: angiologista, cardiologista, dermatologista, neurologista, psiquiatra e reumatologista.

\section{Entre a espera, os meios e o sistema privado: os caminhos percorridos nas RAS}

Inicialmente, identificou-se a percepção das usuárias sobre os motivos que as levaram a procurar a USF. Pôde-se notar que elas perceberam alterações no funcionamento de seu corpo e apresentam necessidades de cuidados a sua saúde. Essas modificações são propulsoras para a procura da USF em busca de respostas para os seus problemas de saúde.

$$
\text { Não sei o que é [...]. (E1) }
$$

Eu quero descobrir o que eu tenho. (E2).

Tenho tosse há mais de um ano e dor de garganta. (E4)

A partir do momento em que as usuárias buscam atendimento na USF e são encaminhadas para outro serviço de atenção à saúde, nota-se que a percepção delas frente ao encaminhamento que as insere em um dos pontos da RAS, se refere às questões que permeiam a temporalidade cabendo, como alternativa, esperar. Isso está representado nos seguintes depoimentos:

eu acho que é de só esperar. Eu queria logo, mas isso aí eu acho que demora. (E1)

é o esperar, é o problema de fila, ou tu estás na fila esperando ou tu marcas e esperas dois, três meses. (E2)

eu fico esperando e nada. (E3) 


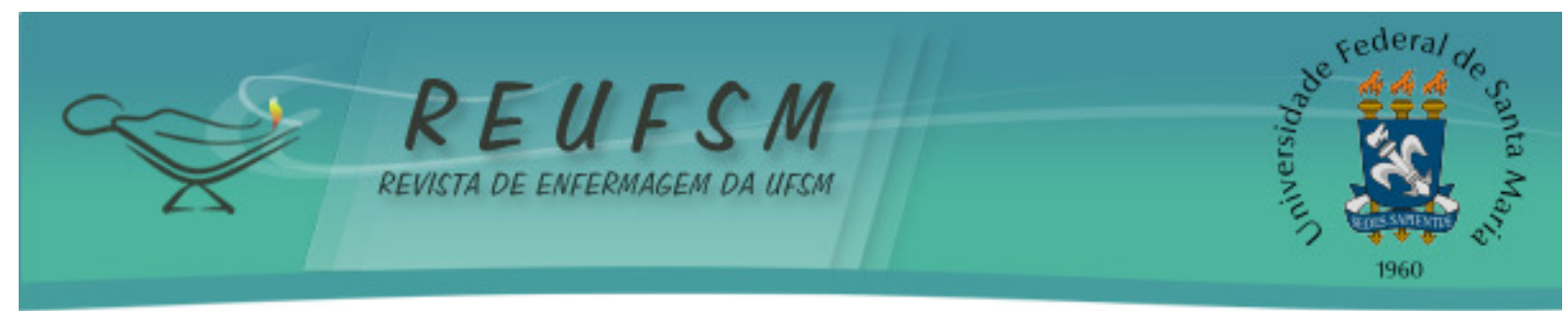

Percebe-se que algumas participantes conhecem a rotina dos encaminhamentos por experiências anteriores, e sabem que terão um longo tempo de espera. Frente a isso e às angustias das usuárias, elas procuram diferentes meios para atender as suas necessidades de saúde de modo mais rápido e efetivo. Dentre eles, a busca pelo sistema privado, o que se evidencia nos depoimentos a seguir:

não tenho condições de pagar, só me resta esperar pelo SUS [...]. Fico muito indignada, isso é descaso com os pobres. Se eu tivesse algum convênio eu não precisava passar por isso, mas só dependo do SUS. (E3)

a não ser que eu que eu pague?! A gente que é pobre tem que depender do SUS. (E1)

para não esperar, tem que pagar particular. (E6)

Além da procura pelo sistema privado, as usuárias não reconhecem o SUS como um direito, pois o destacam como uma alternativa apenas para àquelas pessoas que não podem pagar pelo atendimento no âmbito privado. Outro meio elencado pelas entrevistadas refere-se ao apoio de uma pessoa na USF que facilite o caminho percorrido pelo usuário representando, assim, uma figura de referência e poder. Neste estudo, o coordenador da USF atua desse modo, ao agilizar o processo de encaminhamento:

como o seu (coordenador da USF), esse meio de adiantar as coisas [...]. Eu tinha pressa, ele me ajudou. Aí se esse aqui demorar, vou pedir para ele de novo.(E2)

a não ser no caso do (coordenador da USF) que tem uma autoridade maior [...] tudo foi o (coordenador da USF) que conseguiu para mim, adiantar assim as cirurgias, tudo foi ele que conseguiu, senão eu estaria lá ainda e não teria feito nenhuma cirurgia ainda.(E4)

As percepções referentes à trajetória percorrida nos sistemas de saúde apontam que os caminhos são variados e singulares, sejam eles de espera, de busca por outros serviços de saúde ou por meio da ajuda do coordenador, que exibe poder de facilitar o acesso. Considerar e compreender a diversidade e as peculiaridades envolvidas nesse processo, é uma informação importante para a equipe de saúde, pois pode contribuir no acesso à RAS.

\section{A percepção sobre a atuação dos profissionais de saúde nas RAS}

Quando questionadas sobre as orientações realizadas pelos profissionais de saúde referentes ao encaminhamento realizado, notou-se que existem diversos pontos falhos. Pode-se citar a falta de orientações sobre a RAS, sendo restritas ao tratamento clínico e farmacológico, ocorrendo uma transferência de responsabilidade do profissional para as usuárias.

Nada. Só mandou eu levar ali como agora eu levei, pronto. [...] ele disse que não é da área dele. (E1) 


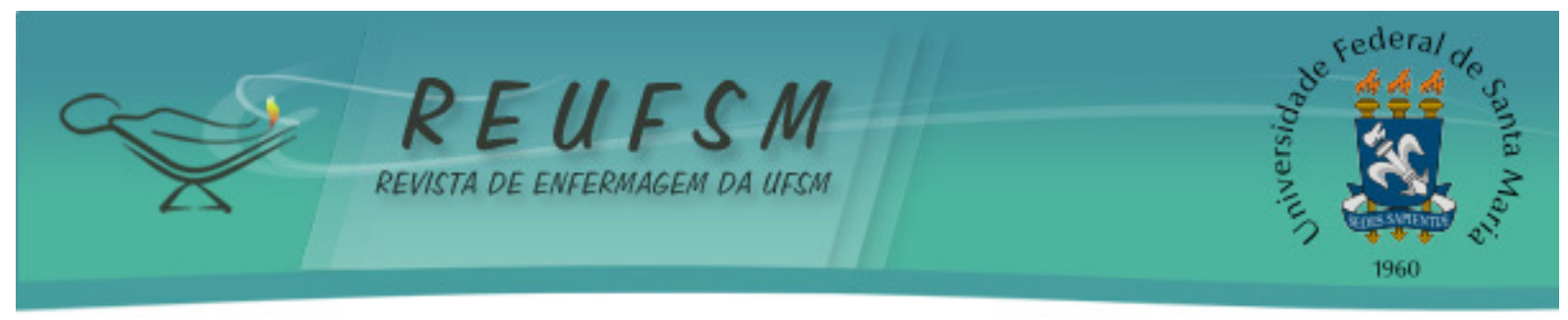

Orientou para que eu continue tomando o remédio para o colesterol que eu tenho. (E2)

Nada, não me disseram nada. A médica só me deu o papel. (E3)

Não, ela disse só: tu perguntas ali na secretaria. (E4)

Percebe-se que, no momento em que o profissional se ausenta da responsabilidade diante do encaminhamento, o resultado é uma prática em saúde fragmentada, ou seja, não integral. Isto pode ter como causa a falta de conhecimento dos profissionais, da gestão e das equipes de saúde da família sobre as RAS. Além disso, as usuárias, quando se referem à unidade, mostram uma percepção referente à estrutura organizacional da USF no que tange, principalmente, à responsabilização sobre o armazenamento e encaminhamento de informações de saúde, inviabilizando os meios disponíveis e possíveis para a resolutividade dos mesmos.

Não, porque se eu venho aqui e pergunto se não foi agendado eles vão dizer: não posso fazer nada [...]. No caso aqui, eu não sei se eles têm como fazer alguma coisa para agendar antes. (E6)

Agora eu vou ali, mas ninguém sabe nada, me passaram para um médico e ele ficou brabo comigo, porque não tinha a receita junto, não me lembrava do nome dos meus remédios para diabete. Eu falei: mas está tudo aí, tem anotado no prontuário, eu sempre consulto aqui, ele me disse que não deram nenhum prontuário para ele [...] perderam o meu encaminhamento, só acontece comigo, não me atendem e ainda perdem o meu encaminhamento. (E3)

Muito mal, muito mal encaminhado [...]. Tu tomas um chá de banco para tu chegares perto da mulher e dizer que tu vieste buscar $o$ encaminhamento. (E5)

Nota-se que impera o sentimento de dúvida e ausência de confiança em relação à equipe devido à falta de informações, empatia e responsabilização pelas demandas dos usuários. A relação mantida entre os usuários e a equipe de saúde, durante o atendimento, se mostrou distante e indiferente, representando uma barreira para o acesso aos serviços de saúde.

\section{DISCUSSÕES}

Pôde-se notar a prevalência do sexo feminino nas buscas pelo sistema de saúde, esse dado também se mostrou presente em um estudo realizado em São Paulo, no qual o número de mulheres atendidas representou a maioria dos casos. ${ }^{9}$ Outra pesquisa na Europa aponta que, quando comparada a presença de homens e mulheres e a procura pelos serviços de saúde, o sexo feminino apresenta maior frequência e familiaridade com as organizações de saúde, pois os homens apresentam algumas barreiras que alteram o envolvimento efetivo na busca pela atenção primária. ${ }^{10}$

No momento em que os usuários se deparam com as dificuldades impostas pelo sistema, sejam elas a dificuldade de acesso ou o tempo de espera frente às longas filas para a consulta com o especialista, esses buscam diferentes meios para conseguir o que 


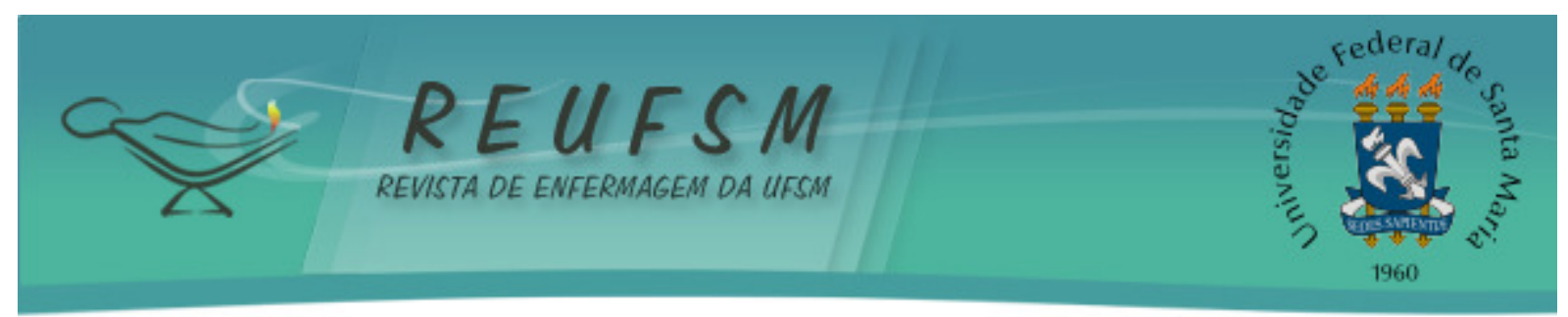

desejam. Todavia, apesar do modelo de saúde já ter passado por consideráveis mudanças, ainda tem influência do modelo biomédico centrado na doença resultando, muitas vezes, em encaminhamentos desnecessários e demasiados. Tal fato reflete no aumento das filas de espera em todos os níveis de densidade tecnológica, além de contribuir para a fragmentação dos cuidados prestados. ${ }^{11}$

A espera, presente em diversos depoimentos se mostrou como um meio utilizado para algumas usuárias quando não encontravam outras opções de acesso à rede. Outras, diante das filas e da espera, buscam como meio capaz de suprir os serviços fragilizados do SUS, aqueles prestados pelo sistema privado de saúde, por apresentar atendimento mais rápido.

Em contrapartida, essas usuárias têm a visão de que o SUS contempla apenas a população sem condições de pagar pelo sistema privado. Esse aspecto demonstra que a representação social sobre o SUS ainda está marcada por uma visão focalizada e assistencialista no atendimento dos segmentos vulneráveis e excluídos da sociedade. ${ }^{12}$ No Brasil, desenvolveu-se a ideia de que política social é para pobres, visão que ocorre paralelamente ao desmonte institucional, orçamentário e da Seguridade Social, resultante da história e dos movimentos políticos e sociais do país. ${ }^{13}$

Ainda, cita-se a presença do coordenador da USF como referência para a população local quanto meio para o acesso rápido e garantido à RAS. Ele atua de forma secundária, não oficial e disponível apenas a um grupo limitado de pessoas, com quem mantêm algum tipo de relação. 0 acesso as RAS torna-se, então, desigual, uma vez que não está disponível da mesma forma para todos.

Pelos diferentes meios de acesso, pode-se inferir que a existência de diferentes portas de entrada no SUS, reflete um cenário em que a entrada no sistema se dá pelo caminho mais fácil ou provável de o usuário ingressar. ${ }^{14}$ Esses pontos de fragilidade remetem à qualidade do acolhimento que vem sendo prestado nas unidades de saúde, referente ao acesso do usuário a esses serviços. Desde quando as usuárias recebem os encaminhamentos e acessam as RAS, percorrendo seus caminhos, nota-se que se sentem distantes do processo no qual estão inseridas, por não saberem qual a próxima etapa dessa trajetória.

Para que o usuário tenha continuidade no cuidado na RAS, é importante destacar o papel da APS como coordenadora do cuidado, em que deve reconhecer os problemas de saúde da população adscrita sob sua responsabilidade, sendo a articuladora entre os demais pontos da rede, promovendo a comunicação para sanar as necessidades e demandas de saúde de cada usuário. ${ }^{3}$ Para isso, é importante que a APS conheça as necessidades de acesso, sejam elas culturais, geográficas, organizacionais ou financeiras, a fim de acolher o usuário, realizar diagnóstico e reordenar recursos para resolver os problemas. $^{3}$

0 acolhimento torna-se, portanto, um modo de executar os processos de trabalho em saúde, ao passo que se estabelece uma relação que permite a identificação dos problemas em contexto mais próximo ao do usuário permitindo, assim, respostas singulares. ${ }^{15,16}$ Ressalta-se que seu objetivo é de prestar um cuidado de qualidade ao usuário e sua família, buscando soluções para cada caso.

Cabe à equipe multiprofissional que atua na APS, na qual está a de enfermagem, prestar cuidados que vão além da técnica, tomando para si a responsabilidade pelos problemas de saúde da população. Com isso, assumir a função resolutiva da APS, visando satisfazer as demandas daquela neste nível de atenção e se responsabilizando pelo acesso aos demais serviços necessários. ${ }^{3}$

A APS deve se constituir como um espaço acessível e flexível para a transformação social. Nesse contexto, o enfermeiro atua otimizando as intervenções de cuidado em saúde, por apresentar capacidade de acolher, identificar as necessidades e expectativas 


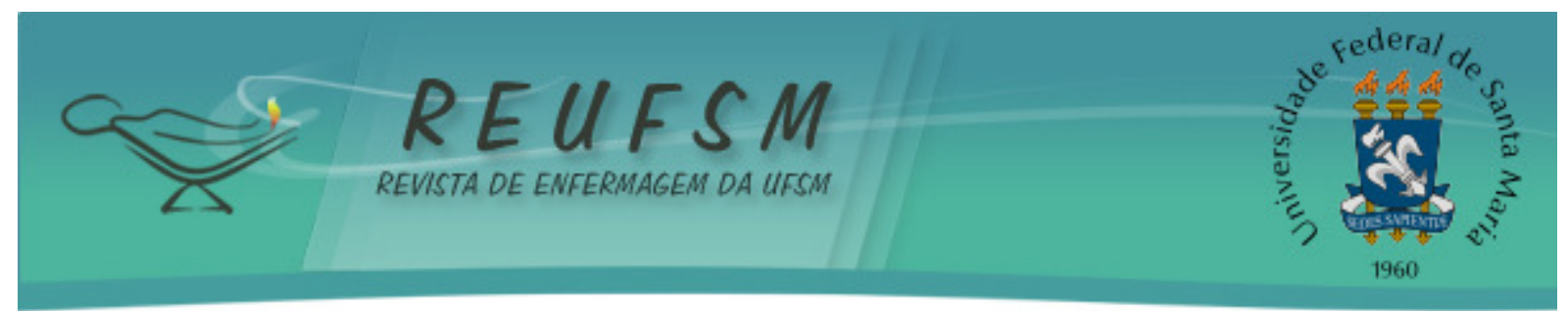

dos usuários, por meio de diálogo direto com esses, considerando tanto os saberes profissionais quanto os dos usuários. ${ }^{17}$

Entretanto, nem sempre essas ações são realizadas e outra pesquisa ressaltou um desamparo dos usuários por parte da unidade e da equipe, referentes às orientações recebidas e à falta de responsabilização do profissional. Os achados indicam que o relacionamento entre equipe e usuários sugere falta de espaço para a participação destes em decisões sobre a conduta terapêutica a ser utilizada, solidificando um processo de submissão que resultou no abandono do tratamento e, consequentemente, na fragmentação do cuidado. ${ }^{18}$

A RAS se constitui em um sistema integrado de saúde, que visa a integralidade do cuidado e institui uma gestão baseada nas necessidades de saúde da população, em que a APS é a coordenadora do cuidado responsável pelo fluxo do usuário. ${ }^{3} 0$ acesso aos serviços de saúde é, portanto, condição para que a continuidade do cuidado seja efetivada, sendo a APS sua coordenadora e, desta forma, a facilitadora para que ele ocorra. ${ }^{19}$

A cartilha da Política Nacional de Humanização (PNH) de 2009, menciona um sistema público de saúde burocratizado e verticalizado, que pouco investe na qualificação dos trabalhadores e dispõe de recursos insuficientes de fomento à cogestão e a valorização e inclusão dos trabalhadores e usuários no processo de produção de saúde. ${ }^{18}$ São citados os problemas atuais que o SUS vem enfrentando, dentre eles, a fragmentação do processo de cuidado e da rede, o que dificulta a complementaridade entre a APS e o sistema de referência. ${ }^{20}$

Outro estudo encontrou essa fragmentação e burocratização na prática cotidiana, propiciando a perpetuação do modelo de atenção, centrado na ação curativa e focado na queixa, o que contradiz a proposta de humanização. ${ }^{21}$ Para se romper com essas ações, a equipe multiprofissional precisa agir para melhoria dos cuidados ao usuário, utilizando uma postura acolhedora, que estabeleça vínculo deles com o serviço, pautado em uma relação de respeito, compreensão e escuta, capaz de oferecer uma resposta resolutiva para a maior parte dos problemas de saúde da população. ${ }^{22}$

Os problemas na acessibilidade podem estar associados à falta de resolubilidade por déficit na formação dos profissionais e no foco ao cuidado integral. A humanização e o acolhimento, embora sejam importantes dispositivos, não resolverão todos os problemas desse nível de atenção para a boa estruturação da RAS. Desse modo, deseja-se que o profissional que atua em qualquer ponto da RAS, se fundamente em princípios técnicos científicos coerentes com os princípios da APS, criando estratégias capazes de interferir no processo de produção de saúde.

Esses fatores podem refletir no cuidado ofertado, no acolhimento dos seus usuários, no estabelecimento de vínculos e na cogestão do processo de saúde e doença. É preciso, então, considerar que, quando ocorrem interações e o usuário é mobilizado, ele é capaz de transformar sua realidade e, desta maneira, se tornar um protagonista como um ator social.

\section{CONCLUSÕES}

Este estudo permitiu identificar que os usuários passam por um longo tempo de espera quando acessam a rede na busca pelo atendimento especializado e, com isso, buscam diferentes meios para consegui-lo. Também se mostrou presente um distanciamento do profissional quanto a sua responsabilização sobre a saúde e a conduta com o usuário, além de uma escuta ineficaz, ressaltando a verticalização existente dentro dessa relação, uma vez que o usuário é mantido à margem das condutas tomadas sobre sua saúde.

Por meio dessas relações, verifica-se a necessidade de qualificação dos profissionais de saúde, principalmente acerca do princípio de integralidade com foco na RAS, para 


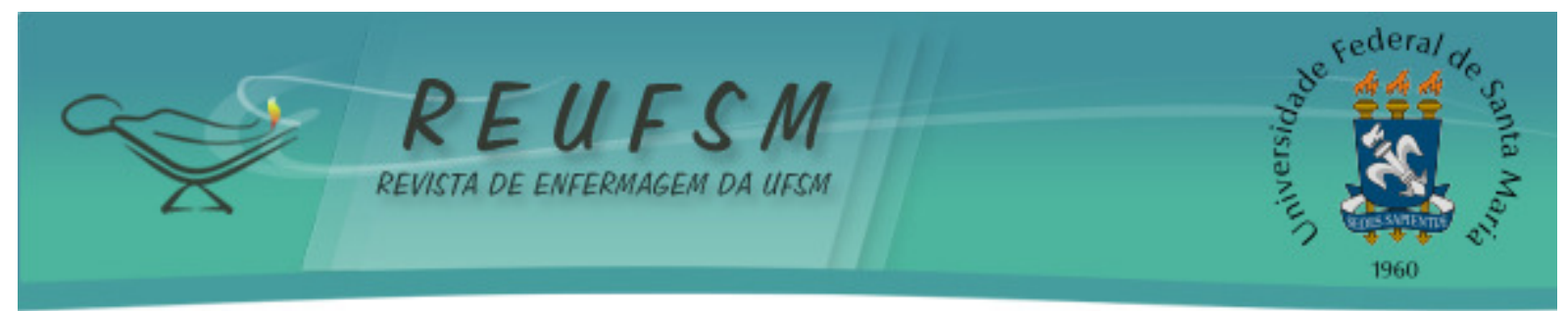

buscar as respostas que os usuários precisam para efetivar os princípios do SUS. Percebese, também, a necessidade da construção de espaços acolhedores como os grupos de saúde participativos nas USF que fomentem o empoderamento dos usuários e sejam formadores de cidadãos conhecedores do SUS, para que possam, a partir de então, atuar com autonomia a fim de exercerem sua cidadania.

Este estudo apresenta limitações relacionadas ao fato de as entrevistas terem sido feitas em um ponto da RAS, por meio da percepção dos usuários. Seria, então, necessário realizarem-se estudos semelhantes em diferentes serviços de saúde e com diferentes atores desses processos, com vistas a conhecer as percepções de RAS sob outras perspectivas. Esse conhecimento pode auxiliar os profissionais e gestores da saúde a refletir e repensar suas práticas, propondo as mudanças necessárias e qualificando o cuidado prestado.

\section{REFERÊNCIAS}

1. Brasil. Ministério da Saúide. Portaria $n^{\circ} 4.279$, de 30 de dezembro de 2010. Estabelece diretrizes para a organização da Rede de Atenção à Saúde no âmbito do Sistema Único de Saúde (SUS). Diário Oficial da União, Brasilia; 2010 fev 31. Seção I, p. 88-93.

2. Mendes EV. As redes de atenção à saúde. Ciênc Saúde Coletiva [Internet]. 2010 [acesso em 2014 out 29];15(5):2297-305. Disponível em: http://www.scielo.br/pdf/csc/v15n5/v15n5a05.pdf.

3. Brasil. Conselho Nacional de Secretários da Saúde. A atenção primária e as redes de atenção à saúde. Brasília: CONASS; 2015. 127 p.

4. Brasil. Ministério da Saúde. Secretaria de Atenção à Saúde. Departamento de Atenção Básica. Política Nacional de Atenção Básica. Brasília: Ministério da Saúde; 2012. 110 p. (Série E. Legislação em Saúde).

5. Serapioni M, Matos AR. Citizen participation and discontent in three Southern European health systems. Soc Sci Med [Internet]. 2014 [acesso em 2016 jan 20];123:1-8. Disponível em: http://www.sciencedirect.com/science/article/pii/S0277953614003657.

6. Ministério da Saúde. Secretaria de Ciência, Tecnologia e Insumos Estratégicos. Departamento de Ciência e Tecnologia. Agenda nacional de prioridades de pesquisa em saúde. 2. ed. Brasília (DF): Ministério da Saúde; 2011. 68 p. (Série B. Textos Básicos em Saúde).

7. Minayo MCS. O desafio do conhecimento: pesquisa qualitativa em saúde. $11^{a}$ ed. São Paulo: Hucitec; 2014.

8. Brasil. Ministério da Saúde. Conselho Nacional de Saúde. Resolução $n^{\circ} 196$, de 10 de outubro de 1996. Aprova diretrizes e normas regulamentadoras de pesquisa envolvendo seres humanos. Brasília: Ministério da saúde/CNS; 1996.

9. Nunes AA,Caccia-Bava MCGG, Bistafa MJ, Pereira LCR, Watanabel MC, Santos V, et al. Resolutividade da Estratégia Saúde da Família e Unidades Básicas de Saúde Tradicionais: Contribuições do PET-Saúde. Rev Bras Educ Méd [Internet]. 2012 [acesso em 2014 nov 05];36(1):27-32. Disponível em: http: / / www.scielo.br/scielo.php?script=sci_arttext\&pid=S010055022012000200004\&lng=en \&nrm=iso. 


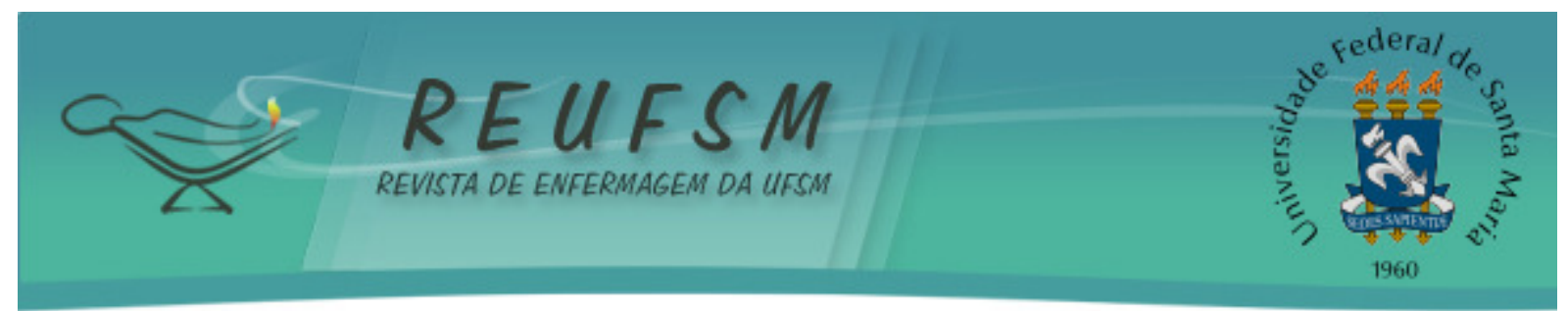

10. Banks I, Baker P. Men and primary care: improving access and outcomes. Trends in Urology and Men's Health [Internet]. 2013 [acesso em 2016 jan 20];4(5):39-41. Disponível em: http://onlinelibrary.wiley.com/doi/10.1002/tre.357/pdf.

11. Reis DO, Araújo EC, Cecílio LCO. Políticas públicas de saúde: Sistema Único de Saúde [Internet]. UNA-SUS/UNIFESP. 2012 [acesso em 2014 out 11]. Disponível em: http://www.unasus.unifesp.br/biblioteca_virtual/esf/2/unidades_conteudos/unidade04/u nidade04.pdf.

12. Martins PC, Cotta RMM, Mendes FF, Priore SE, Franceschinni SCC, Cazal MM, et al. De quem é o SUS? Sobre as representações sociais dos usuários do Programa Saúde da Família. Ciênc Saúde Coletiva [Internet]. 2011 [acesso em 2014 nov 05];16(3):1933-42. Disponível em: http: / / www.scielo.br/scielo.php?script=sci_arttext\&pid=S1413$81232011000300027 \&$ lng=en. http://dx.doi.org/10.1590/S1413-81232011000300027.

13. Paim JS. A Consituição Cidadã e os 25 anos do Sistema Único de Saúde (SUS). Cad Saúde Pública [Internet]. 2013 [acesso em 2017 maio 15];29(10):1927-36. Disponível em: http://www.scielo.br/pdf/csp/v29n10/a03v29n10.pdf.

14. Nery AA, Carvalho CGR, Santos FPA, Nascimento MS, Rodrigues VP. Saúde da família: visão dos usuários. Rev Enferm UERJ [Internet]. 2011 [acesso em 2014 dez 16];19(3):397402. Disponível em: http://www.facenf.uerj.br/v19n3/v19n3a10.pdf.

15. Sena LA, Cavalcanti RP, Pereira IL, Leite SRR. Intersetorialidade e ESF: Limites e Possibilidades no Território de uma Unidade Integrada de Saúde da Família. Rev Bras Ciênc Saúde [Internet]. 2012 [acesso em 2014 out 30];16(3):337-42. Disponível em: http://periodicos.ufpb.br/ojs/index.php/rbcs/article/view/12803/7873.

16. Santos RCA, Miranda FAN. Importância do vínculo entre profissional-usuário na estratégia de saúde da família. Rev Enferm UFSM [Internet]. 2016 [acesso em 2016 nov 07];6(3):350-9. Disponível

https://periodicos.ufsm.br/reufsm/article/view/17313/pdf.

17. Backes DS, Backes MS, Erdmannll AL, BüscherA. O papel profissional do enfermeiro no Sistema Único de Saúde: da saúde comunitária à estratégia de saúde da família. Ciênc Saúde Colet [Internet]. 2012 [acesso em 2015 abr 07];17(1):223-30. Disponível em: http: / /www.scielo.br/scielo.php?script=sci_arttext\&pid=S1413-

$81232012000100024 \&$ Ing=en.http://dx.doi.org/10.1590/S1413-81232012000100024.

18. Schimith MD, Brêtas ACP, Budó MLD, Chiesa AM, Alberti GF. Colonialism in the relationship between workers and service users during care practices: implications for comprehensive care. Esc Anna Nery Rev Enferm [Internet]. 2013 [acesso em $2015 \mathrm{abr}$ 07];17(4):788-95. Disponível em: http://www.scielo.br/scielo.php?pid=S1414$81452013000400788 \&$ script $=$ sci_arttext\&tlng=en.

19. Cunha EM, Giovanela L. Longitudinalidade/continuidade do cuidado: identificando dimensões e variáveis para a avaliação da Atenção Primária no contexto do sistema público de saúde brasileiro. Ciênc Saúde Colet [Internet]. 2011 [acesso em 2017 maio 21];16(1):1029-42. Disponível em: http: / / www.scielo.br/scielo.php?script=sci_arttext\&pid=S1413$81232011000700036 \&$ lng=en. http://dx.doi.org/10.1590/S1413-81232011000700036.

20. Brasil. Ministério da Saúde. Secretaria de Atenção à Saúde. Política Nacional de Humanização. Humaniza SUS: gestão participativa: co-gestão. 2. ed. rev. Brasília: Editora do Ministério da Saúde; 2009. 20 p. (Série B. Textos Básicos de Saúde). 


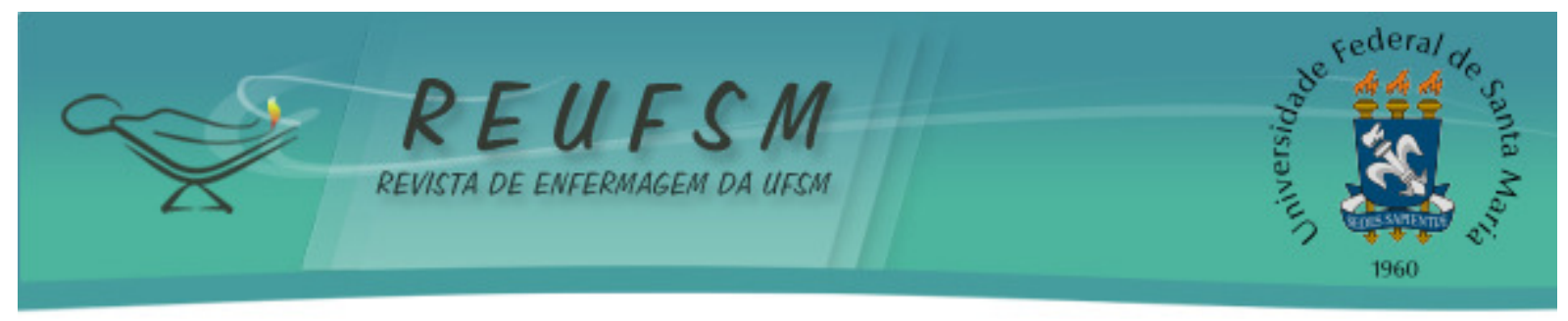

21. Marin MJS, Storniolo LV, Moravcik MY. Humanization of Care from the Perspective of the Family Health Strategy Teams in a City in the Interior of São Paulo, Brazil. Rev Latinoam Enferm [Internet]. 2010 [acesso em 2015 abr 7];8(4):763-9. Disponivel em: http: / / www.scielo.br/scielo.php?script=sci_arttext\&pid=S0104-11692010000400015.

22. Sá SLB, Mattos RR, Pinheiro VL, Pequeno LL, Diógenes MAR. Usuário da estratégia de saúde da família: conhecimento e satisfação sobre acolhimento. Rev Bras Promoç Saúde [Internet]. 2012 [acesso em 2014 maio 21];25(2):96-103. Disponível em: http://ojs.unifor.br/index.php/RBPS/article/view/2250.

Data de recebimento: $13 / 12 / 2016$

Data de aceite: 19/06/2017

Contato do autor principal: Adrielle Chermont da Silva

Endereço Posta: Rua Cel Aníbal G. Barão, 148, ap 303. Centro. Cep: 97050-140. Santa Maria, RS, Brasil.

E-mail: adriellechs@gmail.com 